\title{
A preparative purification process for recombinant Hepatitis B core antigen using online capture by expanded bed adsorption followed by size-exclusion chromatography
}

\begin{abstract}
Hepatitis B core antigen ( $\mathrm{HBcAg}$ ) is an important serological marker used in the diagnosis of hepatitis $\mathrm{B}$ virus (HBV) infections. In the current study, a fast and efficient preparative purification protocol for truncated $\mathrm{HBcAg}$ from Escherichia coli disruptate was developed. The recombinant $\mathrm{HBcAg}$ was first captured by anion exchange expanded bed adsorption chromatography integrated with a cell disruption process. This online capture process has shortened the process time and eliminated the "hold-up" period that may be detrimental to the quality of target protein. The eluted product from the expanded bed adsorption chromatography was subsequently purified using size-exclusion chromatography. The results showed that this novel purification protocol achieved a recovery yield of $45.1 \%$ with a product purity of $88.2 \%$, which corresponds to a purification factor of 4.5 . The recovered $\mathrm{HBcAg}$ is still biologically active as shown by ELISA test.
\end{abstract}

Keyword: Hepatitis B core antigen; Escherichia coli; Online capture process; Expanded bed adsorption chromatography; Size-exclusion chromatography 